\title{
ESTIMATION OF LOS RATES AND ANGLES USING EKF FROM NOISY SEEKER MEASUREMENTS
}

\section{R Ananthasayanam* A K Sarkar** Prashant Vohra** A Bhattacharya** and R Srivastava**}

*Professor, Aerospace Engineering Department, IISc, Bangalore-560012, India, sayanam@aero.iisc.ernet.in ${ }^{* *}$ Scientists, DRDL, Po-Kanchanbagh, Hyderabad-500058, India

\section{ABSTRACT}

This paper deals with estimation of Line Of Sight rates and angles of evader with respect to pursuer from available noisy active seeker measurements. During terminal guidance, on board seeker of pursuer acquires the required measurements which are very noisy, non Gaussian and correlated due to glint, eclipsing and thermal noise. Also there is an aperiodic data loss in the inner gimbal pitch and yaw rate measurement channels due to eclipsing. An Extended Kalman Filter has been used to estimate the Line Of Sight rates and angles along with observer states from seeker measurements under such practical situations. The estimation algorithm has been validated under simulated environment and the results are encouraging. Future research to be carried out is also discussed at the end.

KEY WORDS: Eclipsing Noise, Glint Noise, Seeker Measurements, Extended Kalman Filter

\section{INTRODUCTION}

Air to air combat scenarios represent the most demanding environment for pursuer guidance due to high dynamics of both pursuer and evader. Generally the guidance problem is solved by using well known Proportional Navigation (PN) technique, in which the Line of Sight (LOS) angles and angular rates between the evader and interceptor are used for interception [1]. Active radar seeker is generally used in pursuer as sensor to measure range, range rate, LOS angles and rates between pursuer and evader (Fig. 1). These measured signals are contaminated by high degree of noise due to Eclipsing, Glint, Radar Cross Section (RCS) fluctuation and Thermal noise. The eclipsing effect in a Radio Frequency (RF) seeker is a function of Pulse Repetitive Frequency (PRF) and closing velocity. Due to this effect, in a single PRF seeker, measurements are not available periodically. Moreever these measurements are available in noninertial inner gimbal frame (Fig. 2). In a practical scenario, resultant noise generated due to these cumulative effects is highly non Gaussian and time correlated too. Under these constraints the true LOS rates have to be estimated which will be used for guidance application. The present paper discusses Extended Kalman Filter (EKF) algorithm for real time application to process such type of seeker measurements.

Now we briefly review the earlier work on this real time estimation problem which can be characterized as "Expansion of the scenario" [2] in estimation theory. Though many have worked on this problem, only typical recent papers are reviewed here.

All communications are requested to be aldressed to A K Sarkar a_krishna.sarkar@yahoo.com

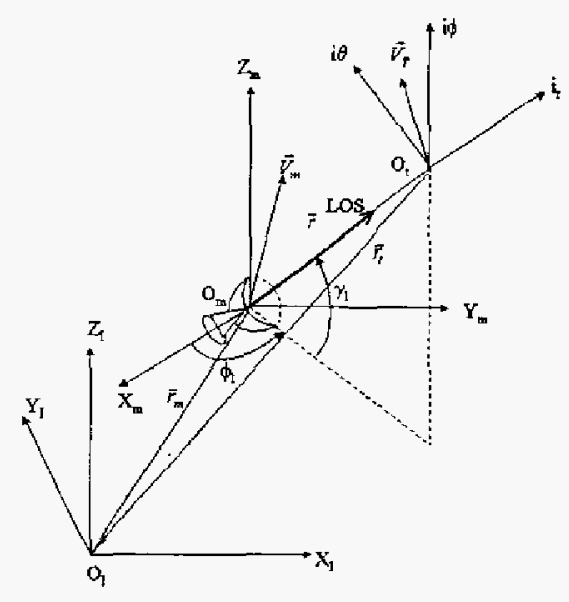

Fig. 1. Schematic Diagram of Pursuer and Evader Engagement

Dowdle et al. [3] estimated target maneuver using EKF where the measurements were obtained by on board active radar seeker. The measurements were relative range, range rate, LOS angles and LOS rates. Balakrishnan [4] and Stallard [5] have estimated LOS rates from passive measurements of LOS angles alone. Based on Stallard's work, Robinson et al. [6] have estimated LOS rates based on relative range, range rate and LOS angles as measurements. Hablani [7] has solved similar problem by reconstructing the LOS rates of exoatmospheric interceptor from LOS angle only measurements. Taur et al. [8] also estimated LOS rates from angle only measurements in two dimensional engagement scenario using EKF. One of the imlportant limitation in the work of these researchers is that in all these cases the measurement angles are azimuth and elevation angle of LOS of evader with respect to pursuer. These formulations are applicable for reconstructing LOS rates from angles when it is tracked by a tracking radar. But seeker measures boresight errors and gimbal angles in gimbal frame. Hence certain orthogonal transformations are necessary between LOS to Inertial and Inertial to Gimbal frame which are not considered in their formulation. Recently Waldman [9] discussed modeling of an imaging seeker and the formulation of an EKF for estimating LOS rates from measurements of relative angular displacement between seeker gimbals and strap down inertial unit. Here he has considered the above transformations in the measurement equations. His aim was to circumvent the need for rate gyro in seeker for which he estimated inner gimbal angular rates relative to pursuer body.

However none of the above work takes into account effect of eclipsing on LOS rates. During the eclipsing period the-mea- 


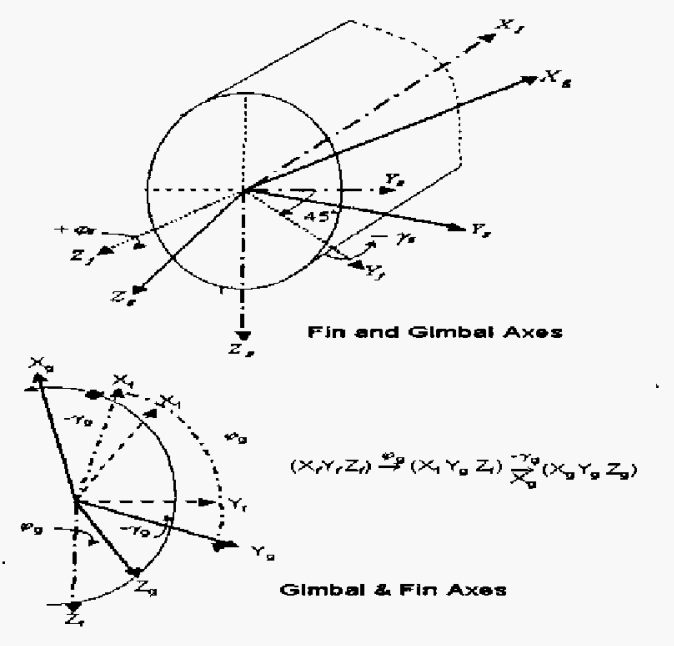

Fig. 2. Different Axes System For Seeker

sured LOS rate data will be lost. No unclassified published literature on LOS rate estimation is available till date to the best of authors' knowledge which accounts for eclipsing phenomenon on measured LOS rates. One more serious problem which is encountered in KF seeker data processing is that the measurement noise in bore sight error consists of two parts, (1) Periodically correlated receiver noise as a function of time varying fluctuating SNR and (2) Glint Noise which is highly non Gaussian depending on evader length, modulation ratio and is time correlated.

In the prosent study, the EKF based estimator has been designed in observer form and available measurements are relative range, range rate, gimbal angles and LOS rates. The relative range and range rates are contaminated by Gaussian noise. The gimbal angles and LOS rates are measured at gimbal frame. They are contaminated by non Gaussian noise due to effect of eclipsing, RCS fluctuation and glint. Also during eclipsing period LOS rates as measurement is not available for processing. These practical constraints will mimic the realistic seeker measurements in closest proximity. Under these constraints an EKF has been designed presently to estimate target relative position, relative velocity and acceleration components as observer states. Based on these state informations, LOS rates and angles have been estimated.

The paper is organized as follows. At first proposed EKF based estimator consisting of both state and measurement model is described. Then a simulation case study of LOS rate estimation based on the proposed model is described. The paper concludes with suggestions for further research.

\section{MATHEMATICAL MODELING OF PRESENT ESTIMATION PROBLEM}

The seeker is a radar inside a moving pursuer which measures relative range $(r)$, range rate $(\dot{r})$, Gimbal angles $\left(\gamma_{s}, \phi_{g}\right)$ and LOS angular rates with respect to inner gimbal frame $\left(\dot{\gamma}_{i g}, \dot{\phi}_{i g}\right)$ in both pitch and yaw plane respectively. These measurements are functions of relative position $(\Delta x, \Delta y, \Delta z)$, relative velocity $\left(\Delta V_{x}, \Delta V_{y}, \Delta V_{z}\right)$ of evader with respect to purster, accelerations of pursuer $\left(a_{m x}, a_{m y}, a_{m z}\right)$ and acceler-

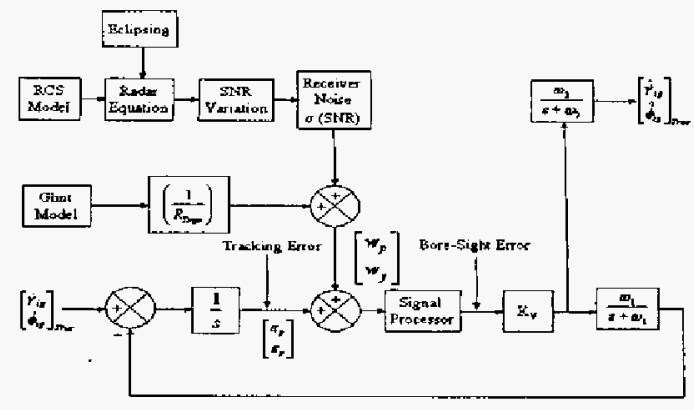

Fig. 3. Schematic Diagram of Seeker Model

ation of evader $\left(a_{t x}, a_{t y}, a_{t z}\right)$ respectively (Fig. 1, Fig. 2). The details of the system plant and the measurement model are given in Appendix. The estimation problem consists nine state variables $\left(\Delta x, \Delta y, \Delta z, \Delta V_{x}, \Delta V_{y}, \Delta V_{z}, a_{t z}, a_{t y}, a_{t z}\right)^{T}$ and six measurements $\left(r, \dot{r}, \dot{\phi}_{g}, \gamma_{g}, \dot{\phi}_{i g}, \dot{\gamma}_{i g}\right)^{T}$ as given by Eqns. (1- $\left.\gamma\right)$ respectively. During eclipsing measurement set will consist of only four measurements $\left(r, \dot{r}, \phi_{g}, \gamma_{g}\right)^{T}$. Total measurements being four or six depending on eclipsing or no eclipsing zone, sequential measurement update is most suitable [13]. The inputs to the state equations are pursuer acceleration components $\left(a_{m x}, a_{m y}, a_{m z}\right)$ and inputs to measurement equations are pursuer attitude quaternion q. In Eqn. 1 the dynamical equations corresponding to target acceleration estimation consists of modeling error which is generally true in practice. So the filter tuning elements are $\left(Q_{a_{t x}}, Q_{a_{l y}}, Q_{a_{t z}}\right)$ and they can be varied by proper combinations of $\left(\sigma_{a_{l s}}, \sigma_{a_{t y}}, \sigma_{a_{\ell x}}\right)$ and $\left(\tau_{x}, \tau_{y}, \tau_{z}\right)$ which are characteristics of the amount of noise and time constant of correlation respectively.

\section{ANALYSIS OF SIMULATION RESULTS}

Now let us estimate the LOS rates and observer states from measured seeker data in a simulated environment. The relam tive range and range rate (one $\sigma$ ) values have been taken as 50 $\mathrm{m}$ and $10 \mathrm{~m} / \mathrm{s}$ respectively. Seeker gimbal angles and LOS rates in gimbal frame have been generated by using tentative seeker model (Fig. 3). Input to the secker model is eclipsing noise, glint noise and noise due to RCS fluctuation. The eclipsing noise of LOS rates in inner gimbal frame $\left(\dot{\phi}_{i g}, \dot{\gamma}_{i g}\right)$ varies with respect to Range to go $\left(R_{\text {to go }}\right)$. It is maximum at seeker lock on range $R_{\max }$. Generally noise standard deviation on LOS rates $\sigma_{\phi_{i g}}=\sigma_{\gamma_{i g}}=\sigma_{\max }\left(R_{t o g o} / R_{\text {rax } x}\right)^{2} \mathrm{deg} / \mathrm{sec}$ where $R_{\text {max }}$ is $10 \mathrm{~km}$ and $\sigma_{\max }$ is $1.8 \mathrm{deg} / \mathrm{sec}$. In Fig. $3\left(w_{y}, w_{p}\right)^{T}$ consists of superimposition of all seeker noise components. A part from these non Gaussian noises, the rate channels and angle channels have been contanninated by Gaussian noise of $1 \mathrm{deg} / \mathrm{s}$ and 0.5 deg standard deviation respectively.

The $\mathrm{R}$ matrix elements for filter design have been selected by inspection of the measurement signals. Looking at the random fluctuation of signal over it's mean, the measurement variance of 'i'-th channel can be calculated as $r_{i i}=\left(\Delta y_{i} / 3\right)^{2}$ where $\Delta y_{i}$ is the maximum dispersion of the noise component over the mean curve. Initial state uncertainty have been taken as 10 $\%$ over the true values and based on that initial state covariance elements have been evolved. For given target maneu- 
ver conditions $\left(\sigma_{a_{t z}}, \sigma_{a_{t y}}, \sigma_{a_{t z}}\right)=(100,100,100)\left(\mathrm{m} / \mathrm{s}^{2}\right)$ and $\left(\tau_{x}, \tau_{y}, \tau_{z}\right)=(10.0,10.0,10.0)(s e c)$ have been found to be adequate for filter tuning.

Based on 20 Monte Carlo (MC) simulation studies the results were studied. This helps in consistency checking of the filter tuning parameters statistically within the $\mathrm{MC}$ derived statistics. The Figs. (5-7) compare estimated and true range, range rate and gimbal angles along yaw plane. In all the figures the estimation error of each measurement is shown and estimates are very close to true values. Time history of noisy LOS rates with eclipsing effect is shown in Fig. 8 . Comparison of filter estimated rates with true values are shown in Fig. 9. It. is seen from the figure that estimated are very close to actual except at terminal phase. The reason behind this can be attributed as follows. The long departures close to endgame show the inadequacy of modeling the states and process noise components to rapidly change the filter gains. It may be noted that the state equations are kinematic and process noise has been assumed to be white Gaussian. But actually the LOS rate measurements have been contaminated by correlated non Gaussian noise. Comparison of estimated target acceleration $\left(\hat{a}_{t x}, \hat{a}_{t y}, \hat{a}_{t z}\right)$ with true values are shown in Fig. 10. It takes roughly 2 seconds for the filter to stabilize and track the true acceleration.

\section{CONCLUSION AND FUTURE ACTIVITIES}

In this paper pursuer evader relative position and velocity components along with evader acceleration in inertial frame have been estimated using EKF from available seeker measurements. Aperiodically the LOS rate data is non available due to eclipsing phenomenon. Though the LOS rates are very noisy consisting of eclipsing, glint and RCS fluctuation noise, present state estimation formulation estimates LOS rates quite accurately. Only during terminal phase the estimated Is OS rate is erroneous with respect to the true value. Future suggested activities are

- Measurements with non Gaussian noise can be processed using EKF based on the approach of Wu [10]. This could definitely improve the estimation error during endgame.

- Present estimator along with different guidance laws are to be integrated with 6 DOF simulation model of pursucr and evader engagement as state observer in close loop to study miss distance performance at interception.

Acknowledgement: Authors thank Dr. S. Gollakota and Mr. $\mathrm{R} N$ Bhattacharjee, scientists of DRDL for projecting the need and providing motivation behind present research.

\section{REFERENCES}

[1] Lin C F: Modern Navigation, Guidance and Control Processing . Volume 2, Prentice Hall (1991).

[2] Ananthasayanam M R: Fascinating Perspectives of State and Parameter Estimation Techniques, Paper No AIAA2000-4319 (2000).

[3] Dowdle J R, Athans M, Gully S, Willasky A S: An Optimal Control and Estimation Algorithm For Missile Endgame Guidance, Proceedings of IEEE Conference on Decission and Control, (1982).

[4] Balakrishnan S N: Extension to Modified Polar Coordinates and Applications with Passive Measurements, Journal of Guidance, Control and Dynamics, Volume 12, No 6, pp 906-912, December (1989).
[5] Stallard D V: An Angle Only Tracking Target For Maneuvering Target, Paper No AIAA-90-3343-CP (1990).

[6] Robinson P N and Yin M R: Modified Spherical Coordinates For Radar, Paper No AIAA-94-3546-CP (1994).

[7] Hablani H B: Pulse Guidance of Exoatmospheric Interceptors With Image Processing Delays in Angle Measurements, Paper No AIAA-2000-4272-CP (2000).

[8] Taur D R and Chern J S: A Modified Proportional Guidance Law For IR. Homing Missiles, Paper No AIAA-2000$4160-\mathrm{CP}$

9] Waldmann J: Line of Sight Rate Estimation and Linearizing Control of an Imaging Seeker in a Tactical Missile Guided by Proportional Navigation, IEEE Transactions on Control System Technology, Volume 10, No 4, pp 556567 ,July (2002).

[10] Wu W R: Target Tracking with Glint Noise, IEEE Transactions on Aerospace and Electronic Systems, Volume 29. No 1, pp 174-185, January (1993).

[11] Borden B H and Mumford M L: A Statistical Glint/Radar Cross Section Target Model, IEEE Transactions on Aerospace and Electronic Systerns, Volume 19, No 5, Pp 781-785, September (1983).

[12] Singer R A: Estimating Optimal Tracking Filter Performance for Manned Maneuvering Targets, IEEE Transactions on Aerospace and Electronic Systemss, Vol. AES-6, No. 4, pp 473-483, July (1970).

[13] Stengel R: Stochastic Optimal Control with theory and applications, John Willey and Sons (1986).

\section{APPENDIX}

\section{A. STATE EQUATIONS}

The state equations are

$$
\begin{array}{ll}
\Delta \dot{x}=\Delta V_{x} & \Delta \dot{y}=\Delta V_{y} \\
\Delta \dot{z}=\Delta V_{z} & \Delta \dot{V}_{y}=a_{t y}-a_{m y}+w_{a_{m y}} \\
\Delta \dot{V}_{x}=a_{t x}-a_{m x}+w_{a_{m x x}} & \cdot \\
\Delta \dot{V}_{z}=a_{t z}-a_{m z z}+w_{a_{m z}} & \dot{a}_{t y}=-\frac{a_{t y}}{\tau_{y}}+w_{a_{t y}} \\
\dot{a}_{t x}=-\frac{a_{t x}}{\tau_{x}}+w_{a_{t x}} & \\
\dot{a}_{t z}=-\frac{a_{t x}}{\tau_{z}}+w_{a_{l, z}} &
\end{array}
$$

Target acceleration components are modeled as first order Gauss Markov [12] with corelation time constant $\left(\tau_{x}, \tau_{y}, \tau_{z}\right)$. Its' random noise component is Gaussian with zero mean $\left(w_{a_{t, r}}(t)\right.$, $\left.w_{a_{t_{y}}}(t), w_{a_{t z}}(t)\right)$ with process noise covariances $\left(Q_{a_{t x}}=2 \sigma_{a_{t r}}^{2} / \tau_{x}\right)$, $\left(Q_{a_{t y}}=2 \sigma_{a_{t y}}^{2} / \tau_{y}\right)$ and $\left(Q_{a_{t z}}=2 \sigma_{a_{t x}}^{2} / \tau_{z}\right)$. Here $\left(\sigma_{a_{t x}}, \sigma_{a_{t y}}, \sigma_{a_{\ell z}}\right)$ are uncertainty in target acceleration components.

\section{B. MEASUREMENT EQUATIONS}

Pursuer Direction Cosine Matrices (DCM) are given below.

DCM of fin wrt body $C_{b}^{f}=\left[\begin{array}{ccc}1 & 0 & \\ 0 & \frac{1}{\sqrt{2}} & \frac{1}{\sqrt{2}} \\ 0 & -\frac{1}{\sqrt{2}} & \frac{1}{\sqrt{2}}\end{array}\right]$

DCM of body wrt Inertial $C_{i}^{b}=$

$$
\left[\begin{array}{l}
\left(q_{4}^{2}+q_{1}^{2}-q_{2}^{2}-q_{3}^{2}\right) 2\left(q_{1} q_{2}+q_{3} q_{4}\right) 2\left(q_{1} q_{3}-q_{2} q_{4}\right) \\
2\left(q_{1} q_{2}-q_{3} q_{4}\right)\left(q_{4}^{2}-q_{1}^{2}+-q_{2}^{2}-q_{3}^{2}\right) 2\left(q_{2} q_{3}+q_{1} q_{4}\right) \\
2\left(q_{1} q_{3}+q_{2} q_{4}\right) 2\left(q_{2} q_{3}-q_{1} q_{4}\right)\left(q_{4}^{2}-q_{1}^{2}-q_{2}^{2}+q_{3}^{2}\right)
\end{array}\right]
$$


Body Quaternion $\mathbf{q}=q_{1} i+q_{2} j+q_{3} k+q_{4}$

DCM of LOS wrt Inertial $C_{i}^{L O S}=$

$$
\left[\begin{array}{lll}
\cos \gamma_{l} \cos \phi_{l} & \cos \gamma_{l} \sin \phi_{l} & \sin \gamma_{l} \\
-\sin \phi_{l} & \cos \phi_{l} & 0 \\
-\sin \gamma_{l} \cos \phi_{l} & -\sin \gamma_{l} \sin \phi_{l} & \cos \gamma_{l}
\end{array}\right]
$$

The measurements along the LOS frame (Fig. 1) are

$$
\begin{aligned}
& r=\sqrt{\Delta x^{2}+\Delta y^{2}+\Delta z^{2}} ; \dot{r}=\frac{\Delta x \dot{\Delta x}+\Delta y \Delta y+\Delta z \dot{\Delta} z}{\sqrt{\Delta x^{2}+\Delta y^{2}+\Delta z^{2}}} \\
& \phi=\tan ^{-1} \frac{\Delta y}{\Delta x} ; \dot{\phi}=\frac{(\Delta x \dot{\Delta y}-\Delta y \Delta x)}{\left(\Delta x^{2}+\Delta y^{2}\right)} \\
& \gamma=\tan ^{-1} \frac{\Delta z}{\sqrt{\Delta x^{2}+\Delta y^{2}}} \\
& \dot{\gamma}=\frac{\dot{\Delta z}\left(\Delta x^{2}+\Delta y^{2}\right)-\Delta z(\Delta x \dot{\Delta x}+\Delta y \Delta y)}{\left(\Delta x^{2}+\Delta y^{2}+\Delta z^{2}\right)\left(\sqrt{\Delta x^{2}+\Delta y^{2}}\right)}
\end{aligned}
$$

The seeker measurements of inner gimbal angles along yaw and pitch plane $\left(\phi_{g}, \gamma_{g}\right)$ and LOS rates along yaw and pitch plane in inner gimbal frame $\left(\dot{\phi}_{i g}, \dot{\gamma}_{i g}\right)$ are assumed to be available in fin frame. The gimbal axes frame with respect to missile body frame is shown in Fig. 2. Now let us transform LOS vector to fin frame to obtain gimbal angles in fin frame.

LOS vector in fin frame $(l, m, n)$ is

$$
\bar{i}_{L O S}^{F_{i n}}=C_{b}^{F i n} C_{i}^{b} C_{L O S}^{i}\left[\begin{array}{l}
1 \\
0 \\
0
\end{array}\right]
$$

The gimbal angles $\left(\phi_{g}, \gamma_{g}\right)$ in fin frame are (Fig. 2)

$$
\phi_{g}=\tan ^{-1}\left(\frac{m}{l}\right) ; \quad \gamma_{g}=\tan ^{-1}\left(\frac{n}{\sqrt{l^{2}+m^{2}}}\right)
$$

The rotation sequence is $\left(x_{f} y_{f} z_{f}\right) \stackrel{\phi_{g}}{\rightarrow}\left(x_{1} y_{g} z_{f}\right) \stackrel{-\gamma_{g}}{\rightarrow}\left(x_{g} y_{g} z_{g}\right)$. So DCM from fin to inner gimbal frame is

$$
C_{f}^{g}=\left[\begin{array}{lll}
\cos \gamma_{g} \cos \phi_{g} & \cos \gamma_{g} \sin \phi_{g} & \sin \gamma_{g} \\
-\sin \phi_{g} & \cos \gamma_{g} & 0 \\
-\sin \gamma_{g} \cos \phi_{g} & -\sin \gamma_{g} \sin \phi_{g} & \cos \gamma_{g}
\end{array}\right]
$$

Now let us convert true LOS rates in LOS frame to obtain true LOS rates in inner gimbal frame.

$$
\begin{aligned}
{\left[\begin{array}{c}
\omega_{g x} \\
\dot{\phi}_{i g} \\
\dot{\gamma}_{i g}
\end{array}\right]=\left[\begin{array}{l}
\omega_{g x} \\
\omega_{g y} \\
\omega_{g z}
\end{array}\right] } & =\vec{\omega}_{g}=C_{f}^{g} C_{b}^{f} C_{i}^{b} C_{l}^{i} \vec{\omega}_{L O S} \\
& =C_{f}^{g} C_{b}^{f} C_{i}^{b} C_{l}^{i}\left[\begin{array}{l}
\dot{\phi}_{l} \sin \gamma_{l} \\
\dot{\hat{\gamma}}_{l} \\
\dot{\phi}_{l} \cos \gamma_{l}
\end{array}\right]
\end{aligned}
$$

The range and range rate equations will be same in LOS or gimbal angle frame. So the seeker measurement equations in gimbal frame are

$$
\begin{array}{lll}
r_{m}=r_{l}+\eta_{1} & \text { Range (Eqn. 2) } \\
\dot{r}_{m}=\dot{r}_{l}+\eta_{2} & \text { Range rate (Eqn. 2) } \\
\phi_{g m}=\phi_{g}+\dot{\eta}_{3} & \text { Yaw plane gimbal angle (Eqn. 4) } \\
\gamma_{g m}=\gamma_{g}+\eta_{4} & \text { Pitch plane gimbal angle (Eqn. 4) } \\
\dot{\phi}_{i g m}=\dot{\phi}_{i g}+\eta_{5} & \text { Yaw LOS rate along gimbal (Eqn. 6) } \\
\dot{\gamma}_{i g m}=\dot{\gamma}_{i g}+\eta_{6} & \text { Pitch LOS rate along gimbal (Eqn. 6) }
\end{array}
$$

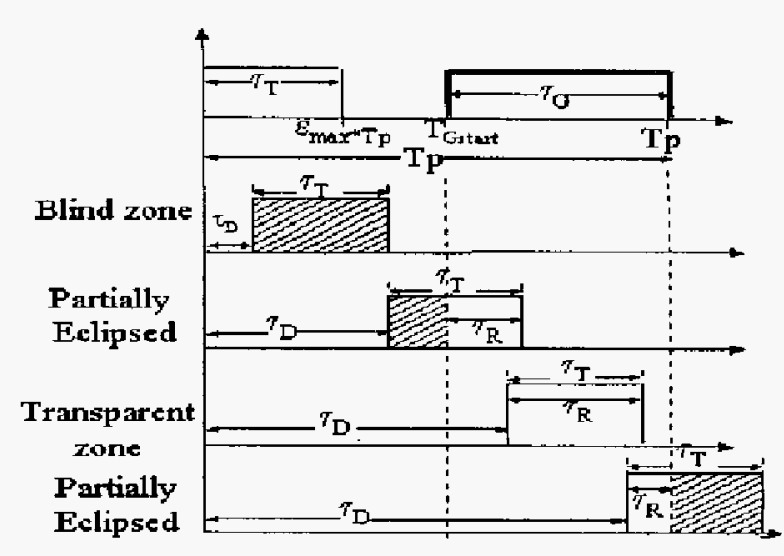

Fig. 4. Eclipsing Phenomenon In Seeker

Here $\left(\eta_{1}, \eta_{2}\right)$ are zero mean Gaussian noise. For gimbal angles $\eta_{3}=\epsilon_{y}+w_{y}$ and $\eta_{4}=\epsilon_{p}+w_{p}$ (Fig. 3).

\section{RECEIVER ANGLE TRACKING ERROR NOISE}

Angle tracking noise is modeled as a Gaussian noise with standard deviation $\sigma_{m}$. This standard deviation of angle tracking noise is a function of Signal to Noise ratio at receiver with relationship $\sigma_{m}=\frac{K_{t}}{\sqrt{S N R}}$. Again SNR can be written as per Radar Range equation $S N R=\frac{K d r^{2}}{R_{T M}^{4}}$ where $d r=\frac{\tau_{R}}{T_{p}}$ is a cyclic time varying quantity varying between 0 to $\varepsilon_{\text {max }}$ due to eclipsing, where $\varepsilon_{\text {max }}$ depends on receiver gate mechanism of a particular seeker. $R_{T M}$ is the instantaneous pursuer evader distance. Eclipsing phenomna is explained in Fig. 4. For no eclipsing case $d r$ can be taken as $\varepsilon_{\max }$. Again combining equations in previous paragraph, we obtain

$$
\sigma_{m}=\frac{K_{1}}{\sqrt{K}} \frac{R_{T M}^{2}}{d r}=K_{2} \frac{R_{T M}^{2}}{d r} .
$$

If $\sigma_{m}=\sigma_{\max }^{o}$ at $R_{\max } K m, K_{2}=\varepsilon_{\max } \frac{\sigma_{\max }}{R_{\max }^{2}}$. For any range to go, angle tracking noise can be expressed as

$$
\sigma=\sigma_{\max }\left(\frac{R_{T A L}}{R_{\max }}\right)^{2} \frac{\varepsilon_{\max }}{d r}
$$

Here $\varepsilon_{\max }=1 / 4, R_{\max }=10 \mathrm{Km}$ and $\sigma_{\max }=1.8^{\circ}$.

\section{SIGNAL PROCESSING BLOCK}

True angle tracking errors $\left(\varepsilon_{y}, \varepsilon_{z}\right)$ are contaminated by receiver noise and glint noise $\left(w_{p}, w_{y}\right)$ and passed through to signal processing block (Fig. 3). Functionality of DSP block assumed to be a mean value of 9 pulses within $10 \mathrm{~ms}$ period. Glint noise model is assumed to be student ' $t$ ' distribution [11]. 


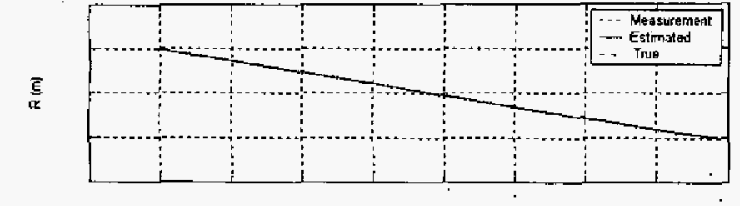

Theme(sec)

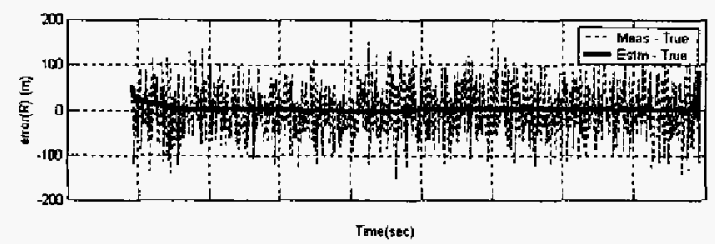

Fig. 5. Comparison of Typical Measured Range with Estimated and True value along with Estimation Error
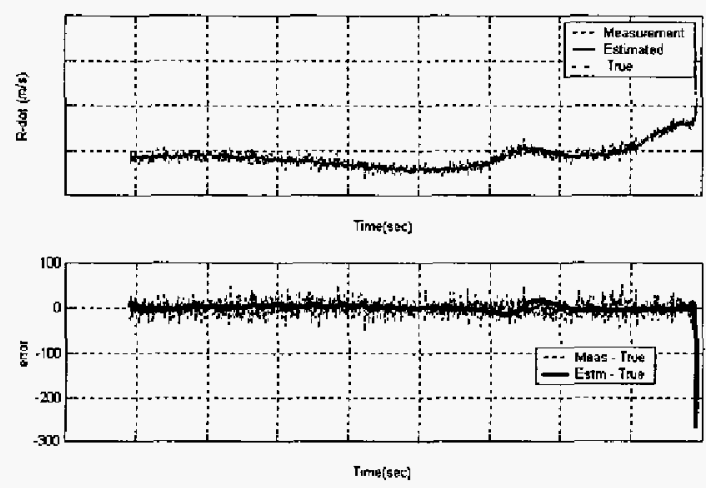

Fig. 6. Comparison of Typical Measured Range Rate with Estimated and True value along with Estimation Error
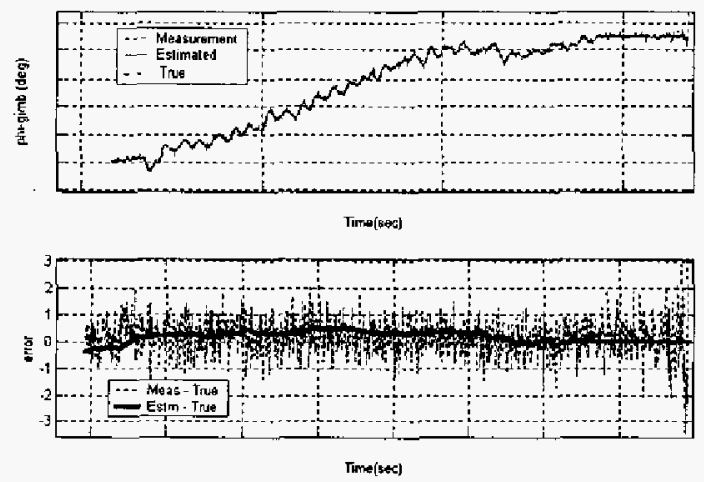

Fig. 7. Comparison of Typical Measured Yaw Gimbal Angle with Estimated and True value along with Estimation Error
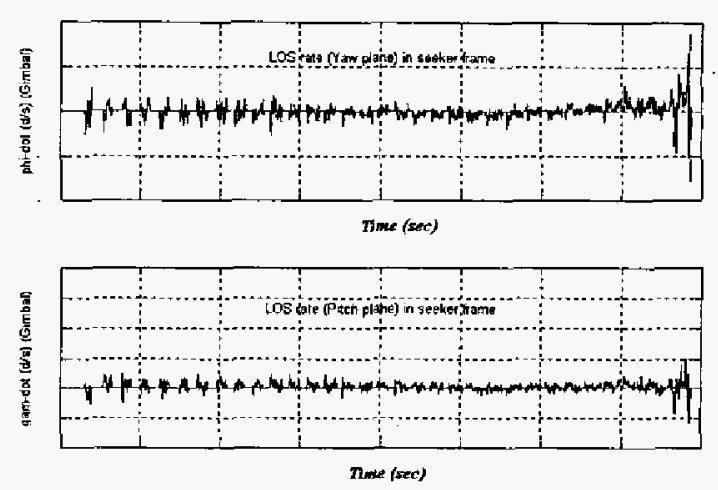

Fig. 8. Measured Typical Yaw and Pitch LOS Rates Time History in Gimbal Erame
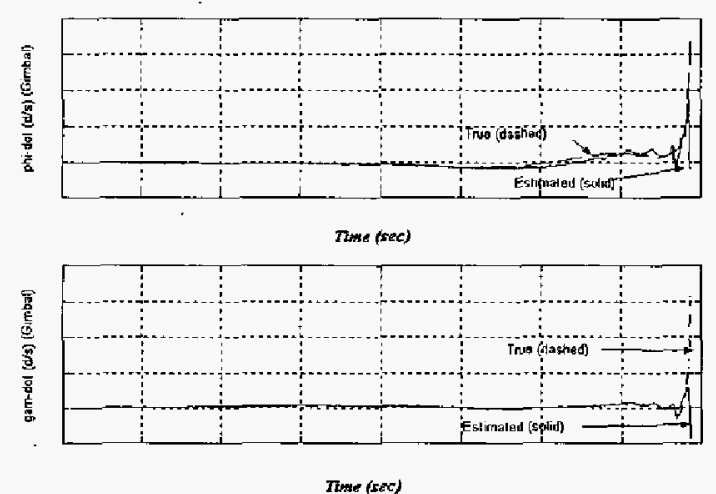

Fig. 9. Comparison of Typical EKF Estimated and True Yaw and Pitch LOS rates time history in Gimbal Frame

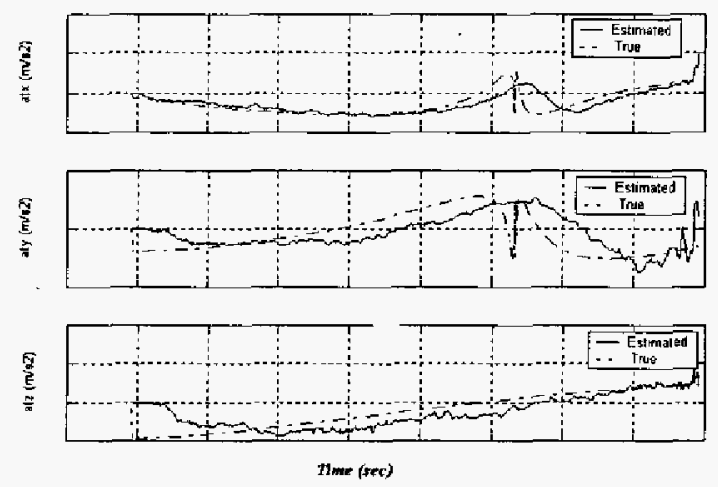

Fig. 10. Comparison of Typical EKF Estimated ( $a_{t x}, a_{t y}$, $\left.a_{t z}\right)\left(m / s^{2}\right)$ with True Value 